\title{
Cold atom realizations of Brownian motors
}

\author{
Ferruccio Renzoni \\ Departement of Physics and Astronomy, \\ University College London, Gower Street, London WC1E 6BT, \\ United Kingdom. E-mail: f.renzoni@ucl.ac.uk. Tel.: 020 7679 7019; Fax. 020 76797145
}

(Dated: October 1, 2018)

\begin{abstract}
Brownian motors are devices which "rectify" Brownian motion, i.e. they can generate a current of particles out of unbiased fluctuations. Brownian motors are important for the understanding of molecular motors, and are also promising for the realization of new nanolelectronic devices. Among the different systems that can be used to study Brownian motors, cold atoms in optical lattices are quite an unusual one: there is no thermal bath and both the potential and the fluctuations are determined by laser fields. In this article recent experimental implementations of Brownian motors using cold atoms in optical lattices are reviewed.

PACS numbers:
\end{abstract}




\section{INTRODUCTION}

Brownian motion is the erratic movement of minute particles immersed in a fluid 1, 2, 3, 4]. The erratic motion is generated by the fluctuating forces acting on the particles, and these forces correspond to the collisions between the immersed particles and the fluid constituents. Obviously the concept of Brownian motion is not limited to particles immersed in a fluid, and the description of this phenomenon applies to all those processes which can be described as the motion of particles in a fluctuating environment.

Brownian motors [5, 6, 7, 8, 9, 10] are devices which "rectify" Brownian motion, i.e. they can generate a current of particles out of unbiased fluctuations. Brownian motors are attracting much interest from researchers from different areas as they seem to have important applications in different fields. In fact, Brownian motors may represent a model for molecular motors, tiny biological engines which transform the energy produced in chemical reactions into unidirectional motion along periodic structures which are macroscopically flat

11]. Furthermore, the mechanism of rectification of fluctuations identified in the study of Brownian motors may lead to new electron pumps, and indeed the study of solid state devices which implement Brownian motors is at present a very active area of research [12, 13, 14].

In this article recent experimental implementations of Brownian motors using cold atoms in optical lattices are reviewed. This is quite an unusual system for a Brownian motor as there is no a real thermal bath, and both the periodic potential for the atoms and the fluctuations are determined by laser fields. With respect to other systems, as for example semiconductor nanostructures, optical lattices offer the significant advantage of being defectfree and highly tunable. In fact, the optical potential is determined by the interference of laser fields, so it has no defects. And both the potential and the fluctuations can be carefully controlled by varying the laser fields' parameters.

The present review consists of two parts. The first part introduces the concept of the Brownian motor, and discusses the limitations imposed by the second law of thermodynamics. Then two examples of Brownian motors are presented: the flashing ratchet and the forced ratchet. The important role of the symmetries is pointed out. The second part of this work describes the implementation of a forced ratchet with cold atoms in an optical lattice. 


\section{BROWNIAN MOTION}

The concept of Brownian motion can be traced back to 1827, when the Scottish botanist Robert Brown observed the ceaseless movement of pollen grains suspended in water. The theoretical description of the phenomenon came much later, with the work of Einstein in 1905 [2], Smoluchowski in 1906 [3] and Langevin in 1908 [4].

The Brownian motion of a macroscopically small but microscopically large particle immersed in a fluid can be simply described in terms of a steady dissipative force and a fluctuating force. For a particle of mass $m$ and position $x(t)$, the equation of motion is

$$
m \ddot{x}(t)=-\gamma \dot{x}(t)+f_{0} \xi(t)
$$

Here $-\gamma \dot{x}(t)$ is the drag force, with $\gamma$ the friction coefficient; and $f_{0} \xi(t)$ is a zero-average fluctuating force, which is generally taken as Gaussian white noise characterised by

$$
\begin{gathered}
\langle\xi(t)\rangle=0 \\
\left\langle\xi(t) \xi\left(t^{\prime}\right)\right\rangle=\delta\left(t-t^{\prime}\right) .
\end{gathered}
$$

The constant $f_{0}$ and the drag coefficient $\gamma$ are not independent, and the fluctuationdissipation relation imposes

$$
f_{0}=\sqrt{2 \gamma k_{B} T}
$$

where $k_{B}$ is the Boltzmann constant. Under these assumptions it is possible to show that the mean-square displacement $\left\langle x^{2}(t)\right\rangle$ evolves at large times as

$$
\left\langle x^{2}(t)\right\rangle=2\left(\frac{k_{B} T}{\gamma}\right) t .
$$

This behaviour is termed normal diffusion, with $D=k_{B} T / \gamma$ the diffusion coefficient.

\section{BROWNIAN MOTORS}

Brownian motors - also called "ratchets" for reasons which will appear clear in the following - are devices which rectify fluctuations producing a current. This is obviously related to the problem of extracting work from fluctuations and therefore, as pointed out by Feynman [15], the second law of thermodynamics imposes strict limitations on the possibility to 
realize Brownian motors. To better illustrate this point, we will refer to two simple devices, shown in Fig. 1 and 2 .

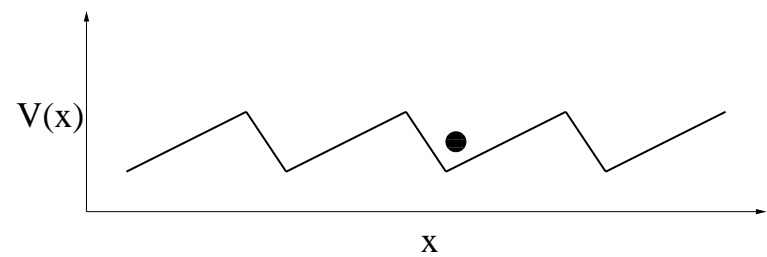

FIG. 1: Brownian particle in a ratchet-potential. The potential is periodic, and has broken spatial symmetry.

Figure 1 shows a Brownian particle, in contact with a thermal bath (not shown) at temperature $T$, in a periodic asymmetric ("ratchet") potential. Intuition would suggest directed motion following the rectification of fluctuations: the thermal fluctuations induce a random walk of the Brownian particle, with the asymmetric potential favouring the motion in one direction, acting in this way as a mechanical diode. However this picture is not correct, and the second law of thermodynamics forbids the generation of a current. Indeed the generation of a current through the periodic potential would imply the possibility of doing work with just one source of heat at a given temperature, which is forbidden by the second law of thermodynamics. Therefore, in spite of the broken spatial symmetry no preferential direction for the motion arises.

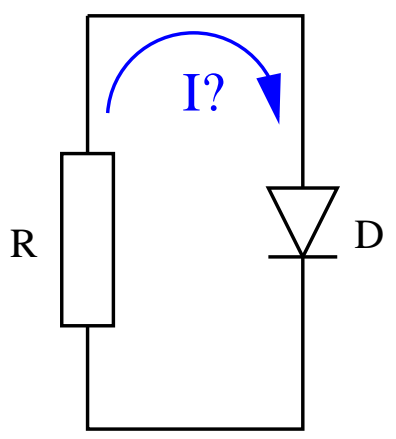

FIG. 2: Circuit composed of a resistor and a diode, illustrating the Brillouin paradox.

Another interesting device is shown in Figure 2, and illustrates the "Brillouin paradox" 16]. This device consists of a resistor connected to a diode. The basic idea is very simple. 
The random thermal motion of the electrons in the resistor results in a fluctuating electromotive force across the resistor, the amplitude of the fluctuations being proportional to the temperature $T$ of the resistor and its resistance $R$. In other words, the resistor $R$ acts as a noise source. The second component of the circuit is a rectifier (electrical diode), connected in series with the resistor. At first sight it may seem that the diode would rectify the fluctuating voltage across the resistor and produce a directed current through the circuit. However this is not possible if the resistor and the diode are at the same temperature. As for the case examined previously, the generation of a directed current through the circuit would imply the possibility of doing work with just one source of heat at a given temperature, which is forbidden by the second law of thermodynamics.

At this point one may wonder whether it is possible at all to realize a Brownian motor, i.e. a device which rectifies fluctuations producing a direct current. The answer is affirmative, provided that the second law of thermodynamics is not violated, i.e. provided that the rectification of fluctuations does not correspond to the extraction of work from just one source of heat at one temperature. As we will see, this can be realized by driving a system out of equilibrium. We will discuss now two examples of Brownian motors realized in this way: the flashing ratchet and the forced ratchet.

\section{A. The flashing ratchet}

The working principle of the flashing ratchet [17] is illustrated in Fig. 3, A sample of Brownian particles experiences an asymmetric potential, analogous to the system described in the previous Section and illustrated in Fig. 1. The difference between the present system and the device of Fig. 1 is that now the potential is intermittent. Indeed the potential is turned on and off repeatedly, either periodically or at random instants. The working prionciple is quite simple (see Fig. 3).

Consider an initial situation with the potential turned on and the Brownian particles localized at the bottom of a given well. Then the potential is turned off, and the Brownian

particles will symmetrically diffuse in space. Then the potential is turned on again, and the Brownian particles are retrapped in both the original well and in a few neighbouring ones. However, as the potential is asymmetric the retrapping will lead to an asymmetric situation, with the number of particles trapped in the wells at the left of the original well different from 


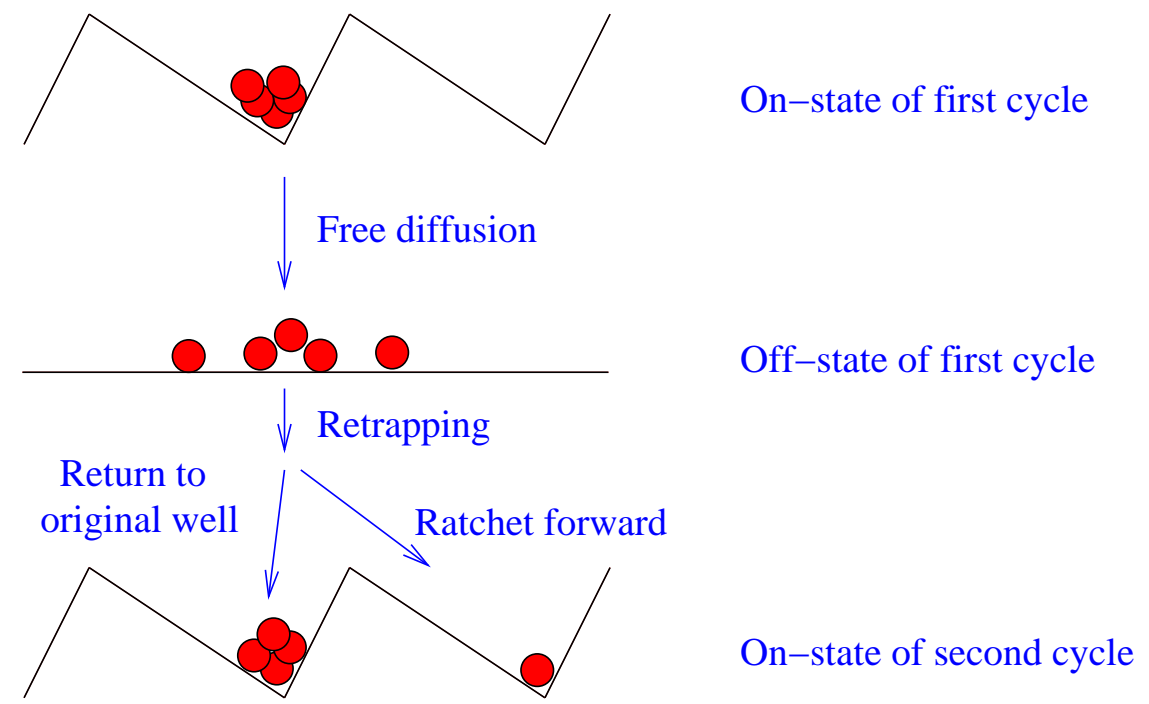

FIG. 3: Working principle of the flashing ratchet.

the number of particles trapped in the wells at the right of the starting location. Indeed it is clear from Fig. 3 that the wells closer to the "steep wall" of the starting well will collect more particles during the retrapping phase. In this way the center of mass of the particle cloud will move, and directed motion is thus obtained.

It is important to point out why the operation of the flashing ratchet does not violate the second law of thermodynamics. This is because work is done on the system while turning on the potential. Thus, although fluctuations are rectified and a current is generated, this does not imply that work has been extracted out of just one heat source as some additional work was necessary to turn on the potential. Therefore the second law of thermodynamics is not violated.

A final remark on the efficiency of the flashing ratchet. A detailed analysis of the dependence of the current amplitude on the flashing ratchet parameters is beyond the scope of the present article, and we refer the interested reader to Refs. [6, 7].

\section{B. The forced ratchet}

A second example of Brownian motor is the forced (or rocking) ratchet (Fig. 41) 18, 19].

Consider once again a sample of Brownian particles in an asymmetric potential, $U_{0}$, with parameters as in Fig. 4. The potential is now rocked by an applied force $F$ fluctuating 

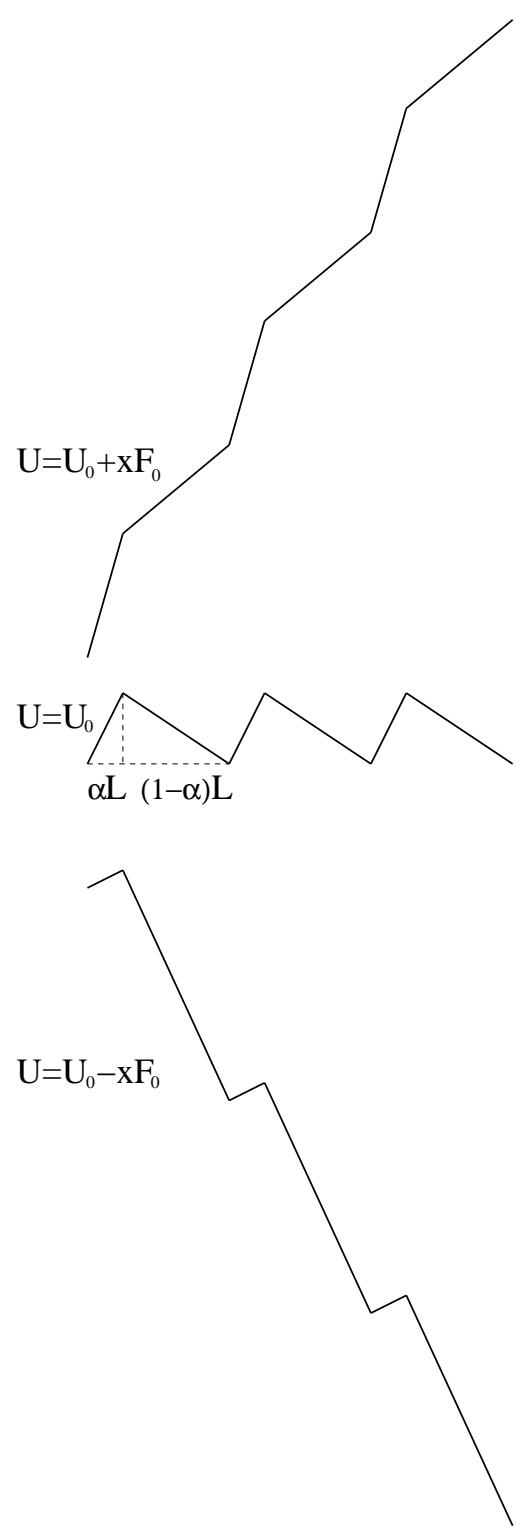

FIG. 4: Forced ratchet potential.

between $-F_{0}$ and $+F_{0}$ with, say, $F_{0}>0$. The relevant parameters to which compare $F_{0}$ are the two slopes of the piecewise potential $U_{0}: \Delta U /[(1-\alpha) L]$ and $\Delta U /[\alpha L]$. Two different cases are relevant for the present analysis. First we examine the case for $\Delta U /[(1-\alpha) L]<F_{0}<$ $\Delta U /[\alpha L]$. This is the situation represented in Fig. 4. When the applied force equals $-F_{0}$ the potential $U=U_{0}+x F_{0}$ increases monotonically for increasing $x$, while when the applied force equals $+F_{0}$ the total potential $U=U_{0}-x F_{0}$ still shows potential minima where the particles can be trapped. It appears evident that a force slowly oscillating between $-F_{0}$ and $+F_{0}$ will lead to directed motion as for $F=-F_{0}$ the potential is monotonically increasing 
while for $F=+F_{0}$ the potential shows wells which allow the trapping of particles. A current in the negative direction is thus produced. We note however that this current is produced also in the absence of fluctuations, and therefore the current cannot be attributed entirely to the rectification of fluctuations.

A more relevant case for the Brownian motors is the subthreshold regime $F_{0}<\Delta U /[(1-$ $\alpha) L]$. In this case the rocking potential alone is not sufficient to induce directed motion. However the presence of some thermal noise allows the generation of a current of particles, and the device acts therefore as a fluctuations rectifier. In this case the current is entirely due to the rectification of fluctuations, and the rocking ratchet is a realization of a Brownian motor.

\section{The rocking ratchet: role of the symmetries}

In the previous Sections we have seen two examples of Brownian motors, both based on an asymmetric ("ratchet") potential. We should mention here that the term "ratchet" is often used as synonymous of Brownian motor, or fluctuations rectifier, even if, as we will see in this Section, Brownian motors can also be realized by using spatially symmetric potentials [20, 21, 22, 23, 24].

In this Section we identify the conditions to observe directed motion in a rocking ratchet, pointing out the role of the symmetries. This will constitute the basis for the cold atom realization of a Brownian motor described in the following Sections.

For the sake of simplicity, we limit our analysis of the role of the symmetries to the case of weak damping of the particles. We refer the interested reader to Refs. [22, 23, 25] for a discussion of the modification of the symmetries of a ratchet-device with increasing dissipation.

Omitting completely the dissipation term, the equation of motion for the particle of mass $m$ can be written as:

$$
m \ddot{x}(t)=-U^{\prime}(x)+E(t) .
$$

Here $U$ is a spatially periodic potential of period $\lambda: U(x)=U(x+\lambda)$, and $E$ a zero mean ac field of period $T: E(t)=E(t+T)$.

Now, we are interested in determining the symmetries which forbid the appearance

of directed motion. These correspond to the transformations in $x, t$ which produce a 
change in sign of the momentum $p$, i.e. the transformations which map a trajectory $\left\{x\left(t ; x_{0}, p_{0}\right), p\left(t ; x_{0}, p_{0}\right)\right\}$, with $x_{0}, p_{0}$ the initial position and momentum, into another one with opposite momentum. These transformations consist of reflections and shifts in time and space and are [21, 22, 23, 25]:

$$
\begin{gathered}
\hat{S}_{a}\left(\begin{array}{c}
x\left(t ; x_{0}, p_{0}\right) \\
p\left(t ; x_{0}, p_{0}\right)
\end{array}\right)=\left(\begin{array}{c}
-x\left(t+T / 2 ; x_{0}, p_{0}\right)+2 \chi \\
-p\left(t+T / 2 ; x_{0}, p_{0}\right)
\end{array}\right) \\
\hat{S}_{b}\left(\begin{array}{l}
x\left(t ; x_{0}, p_{0}\right) \\
p\left(t ; x_{0}, p_{0}\right)
\end{array}\right)=\left(\begin{array}{c}
x\left(-t+2 \tau ; x_{0}, p_{0}\right) \\
-p\left(-t+2 \tau ; x_{0}, p_{0}\right)
\end{array}\right)
\end{gathered}
$$

with $\chi$ and $\tau$ constants. Clearly, if the equation of motion Eq. 5 is invariant under the transformations $\hat{S}_{a}, \hat{S}_{b}$ then no current can be generated. Whether $\hat{S}_{a}, \hat{S}_{b}$ are symmetries of the system depends on the form of $U(x)$ and $E(t)$. It can be shown that [21, 22, 23, 25]:

A) If $U^{\prime}(x+\chi)=-U^{\prime}(-x+\chi)$ and $E(t)=-E(t+T / 2)$ then the equation of motion Eq. 5 is invariant under $\hat{S}_{a}$, and no directed motion can be observed;

B) If $E(t+\tau)=E(-t+\tau)$ the system is invariant under the transformation $\hat{S}_{b}$ and no current can be generated.

In conclusion, the possibility to generate a current is closely related to the symmetries of the system. In the remaining of this paper we will present explicit examples of how the breaking of the relevant symmetries leads to the generation of a current.

\section{The spatially symmetric rocking ratchet}

In the following we will present in detail a cold atom realization of a spatially symmetric rocking ratchet. It is therefore interesting to analyze in the present context the role of the symmetries in that specific case.

We consider here a spatially periodic potential

$$
U(x)=-\cos x
$$

and a bi-harmonic periodic driving

$$
E(t)=E_{1} \cos (\omega t)+E_{2} \cos (2 \omega t+\phi)
$$


If $E_{1} \neq 0$ and $E_{2} \neq 0$ the symmetry $\hat{S}_{a}$ is broken as $E(t) \neq-E(t+T / 2)$, independently of the value of $\phi$. On the other hand, always under the assumption that $E_{1} \neq 0$ and $E_{2} \neq 0$, the symmetry of the system under the transformation $\hat{S}_{b}$ is controlled by the relative phase $\phi: \hat{S}_{b}$ is a symmetry of the system if $\phi=n \pi$, with $n$ integer. To summarize, in the presence of both harmonics the symmetry of the system is controlled by the relative phase $\phi$, and indeed it can be shown [22] that a current appears, whose magnitude $C$ is proportional to

$$
C \propto \frac{E_{1}^{2} E_{2}}{\omega^{3}} \sin \phi
$$

We notice that the current vanishes when $\phi=n \pi$, as the symmetry $\hat{S}_{b}$ is restored.

We stress that the system considered here is purely Hamiltonian, and neither damping nor fluctuations were included in the model. The current is the result of non-linear mixing of the two harmonics, and does not correspond to the realization of a Brownian motor. Theoretical work already examined this system in the presence of dissipation [22]. In the present work we do not repeat the theoretical derivation, and follow a less formal approach: the role of dissipation will be discussed in next Section, where experimental realizations of the rocked ratchet will be reported and it will be shown how the addition of fluctuating forces modifies the picture discussed in the present Section and leads to the realization of a Brownian motor.

\section{COLD ATOM REALIZATION OF A BROWNIAN MOTOR}

The first experiment on the ratchet effect using cold atoms in an optical lattice was reported by Mennerat-Robilliard et al [26]. In that work directed motion was observed in a spatially asymmetric dark optical lattice. In the present paper we describe in detail a different cold atom realization of a Brownian motor, with a forced ratchet realized by using atoms in a bright optical lattice. This is a direct implementation of the ideas discussed in Secs. IIIC and IIID, Before entering into the details of the realization of the forced ratchet, we summarize the basics of bright optical lattices and the underlying Sisyphus cooling mechanism. For more comprehensive reviews of optical lattices, we refer the reader to Refs. [27, 28, 29]. 


\section{A. Bright optical lattices}

Optical lattices are periodic potentials for atoms created by the interference of two or more laser fields. In near-resonant optical lattices a set of laser fields produce at once the periodic potential for the atoms and the cooling mechanism, named Sisyphus cooling, which decreases their kinetic energy so that they are finally trapped at the bottom of the potential wells. We describe here the principles of these optical lattices in the case of a one-dimensional configuration and a $J_{g}=1 / 2 \rightarrow J_{e}=3 / 2$ atomic transition. This is the simplest configuration in which Sisyphus cooling takes place.

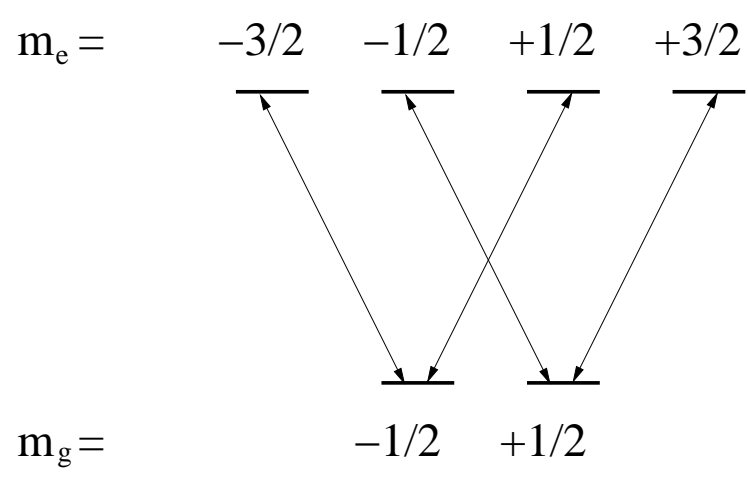

FIG. 5: Atomic level scheme for a $J_{g}=1 / 2 \rightarrow J_{e}=3 / 2$ transition. The arrows indicate the couplings due to $\sigma^{+}, \sigma^{-}$laser excitation.

Consider a transition $J_{g}=1 / 2 \rightarrow J_{e}=3 / 2$ (Fig. 5) coupled to two laser fields with the same amplitude and the same wavelength $\lambda$, linearly polarized and counterpropagating. These laser fields are detuned below atomic resonance and have orthogonal linear polarization (lin $\perp$ lin configuration, see Fig. 6(a)):

$$
\begin{gathered}
\vec{E}_{1}(z, t)=\frac{1}{2} \vec{\epsilon}_{x} E_{0} \exp [i(k z-\omega t)]+c . c \\
\vec{E}_{2}(z, t)=\frac{1}{2} \vec{\epsilon}_{y} E_{0} \exp [i(-k z-\omega t+\alpha)]+c . c
\end{gathered}
$$

where $k=2 \pi / \lambda$ and $\omega=k c$ are the laser field wavevector and angular frequency, respectively. The total electric field is

$$
\vec{E}_{1}(z, t)+\vec{E}_{2}(z, t)=\left[E_{+}(z) \vec{\epsilon}_{+}+E_{-}(z) \vec{\epsilon}_{-}\right] \exp (-i \omega t)+\text { c.c. }
$$


where $\vec{\epsilon}_{ \pm}$are the unit vectors of circular polarization and, after elimination of the relative phase $\alpha$ through an appropriate choice of the origin of the space- and time-coordinates, $E_{+}$ and $E_{-}$are given by:

$$
\begin{gathered}
E_{+}=-i \frac{E_{0}}{\sqrt{2}} \sin k z, \\
E_{-}=\frac{E_{0}}{\sqrt{2}} \cos k z .
\end{gathered}
$$

The superposition of the two laser fields $E_{1}, E_{2}$ produces therefore an electric field characterized by a constant intensity and a spatial gradient of polarization ellipticity of period $\lambda / 2$, as shown in Fig. [6).

(a)

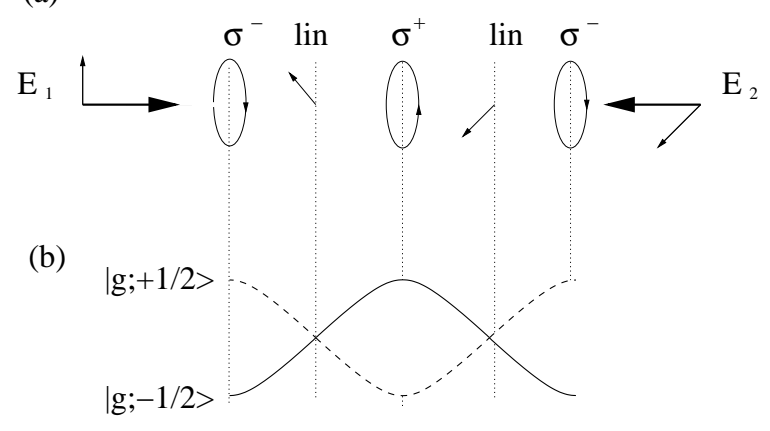

FIG. 6: (a) Arrangement of laser fields in the so-called lin $\perp$ lin configuration, and resulting gradient of ellipticity. (b) Light shift of the two ground-state Zeeman sublevels $|g, \pm 1 / 2\rangle$.

We examine now the effects that the laser fields have on the atoms. The basic mechanism responsible for the generation of a periodic potential is the "light shift": a laser field coupling a given transition, and characterized by an intensity $I_{L}$ and detuning $\Delta$ from atomic resonance, leads to a shift of the ground state energy ("light shift") proportional to $I / \Delta$.

In the present case of a $J_{g}=1 / 2 \rightarrow J_{e}=3 / 2$ transition there are two laser fields coupling each ground state sublevel to the excited state, and contributions from all these couplings have to be taken into account to derive the light shifts $U_{ \pm}$for the ground state Zeeman sublevels $|g, \pm 1 / 2\rangle$. We will omit here the details of the calculations, and simply report the final results for the light shifts (see Ref. [29] for the derivation):

$$
U_{+}=2 \hbar \Delta_{0}^{\prime}\left(\frac{I^{+}}{I}+\frac{I^{-}}{3 I}\right),
$$




$$
U_{-}=2 \hbar \Delta_{0}^{\prime}\left(\frac{I^{-}}{I}+\frac{I^{+}}{3 I}\right)
$$

Here $I^{ \pm}=\left|E^{ \pm}\right|^{2}$ are the intensities of the right- and left-polarization components of the light, and $I=I^{-}+I^{+}$is the total intensity. The quantity $\Delta_{0}^{\prime}$ is the light shift per beam, equal to

$$
\Delta_{0}^{\prime}=\Delta \frac{\Omega_{1}^{2} / 4}{\Delta^{2}+\Gamma^{2} / 4}
$$

where $\Delta$ is the detuning of the optical field from atomic resonance, $\Gamma$ the linewidth of the atomic transition and $\Omega_{1}$ the resonant Rabi frequency for a transition having a ClebschGordan coefficient equal to 1 . The square of the resonant Rabi frequency is proportional to the light intensity, so in the limit of not too small detuning $\Delta$, we find that the light shift per beam scales as $I / \Delta$, as already mentioned. By substituting the expressions (12) for $E^{+}$, $E^{-}$, the light shifts $U_{ \pm}$can be rewritten as:

$$
U_{ \pm}=\frac{U_{0}}{2}[-2 \pm \cos k z]
$$

with

$$
U_{0}=-\frac{4}{3} \hbar \Delta_{0}^{\prime}
$$

the depth of the potential wells. We therefore conclude that the light ellipticity gradient produces a periodic modulation of the light shifts of the ground state Zeeman sublevels (Fig. 6(b)). These periodic modulation acts as an optical potential for the atoms, and indeed these periodically modulated light shifts are usually referred to as optical potentials.

We turn now to the analysis of the cooling mechanism, the so-called Sisyphus cooling [30], which decreases the kinetic energy of the atoms and allows their trapping at the bottom of the wells of the optical potential.

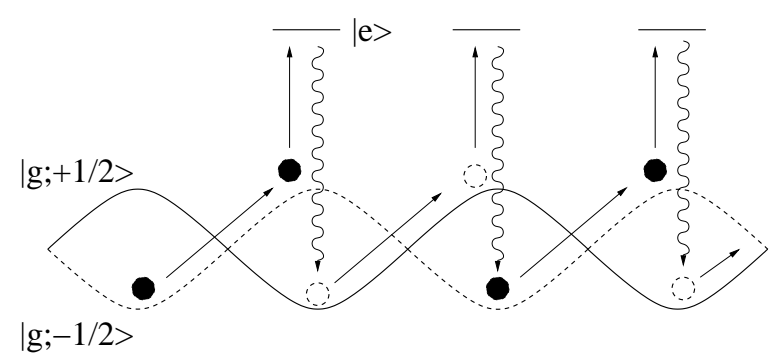

FIG. 7: Sisyphus cooling mechanism. 
Sisyphus cooling is determined by the combined action of the light shifts and of optical pumping, which transfers, through cycles of absorption/spontaneous emission, atoms from one ground state sublevel to the other one. This is illustrated in Fig. [7. Consider an atom moving with a positive velocity, and initially at $z=0$ in the state $|g,-1 / 2\rangle$. While moving in the positive $z$ direction the atom climbs the potential curve corresponding to its actual internal state. This has two consequences: first, a part of the kinetic energy of the atoms is transformed in potential energy; second, the component $\sigma^{+}$of the light increases, which implies the increase of the optical pumping rate towards the level $|g,+1 / 2\rangle$, i.e. an increase of the probability of transfering the atom from the actual internal state $|g,-1 / 2\rangle$ to the state $|g,+1 / 2\rangle$. At the top of the potential hill $(z=\lambda / 4$, see Fig. [6) the polarization of the light is purely $\sigma^{+}$, and the probability to transfer the atom into the sublevel $|g,+1 / 2\rangle$ is very large. The transfer of the atom into the level $|g,+1 / 2\rangle$ results into a loss of potential energy, which is carried away by the spontaneously emitted photon. This process is repeated several times, until the atom does not have enough energy any more to reach the top of a potential hill, and it is trapped in a well. We notice here the analogy with the myth of Sisyphus, king of Corinth, condemned forever to roll a huge stone up a hill which repeatedly rolls back to the bottom before the summit is reached. This is why the described cooling mechanism has been named Sisyphus cooling. The described cooling process leads to the localization of the atoms at the bottom of the potential wells, and we obtain in this way an optical lattice: an ensemble of atoms localized in a periodic potential. We notice that the atoms are localized at the sites where their interaction with the light is maximum. It is because of this property that optical lattices of this type are termed bright optical lattices.

\section{B. Realization of the forced ratchet}

The realization of a forced ratchet requires essentially three elements. First, a periodic potential; second, a fluctuating environment which results in friction and in a fluctuating force. Finally, it should be possible to apply a zero-mean ac-force to the particles (the atoms in the present case). All these requirements can be satisfied by using cold atoms in optical lattices, as it was demonstrated in Ref. [31]. In that work the one-dimensional lin $\perp$ lin optical lattice described in Sec. IVA was taken as periodic potential. We stress that such an optical lattice is spatially symmetric, and indeed the work of Ref. [31] aimed to realize the spatially 
symmetric rocking ratchet introduced in Sec. IIIC. We turn now to the analysis of the friction and fluctuations in the optical lattice, the second element necessary to use optical lattices as a model system for Brownian motors. As already discussed, the optical pumping between the different atomic ground state sublevels combined with the spatial modulation of the optical potential leads to the cooling of the atoms and to their localization at the minima of the optical potential. The essential fact for the realization of Brownian motors is that even after the cooling phase, charaterized by a decrease of the kinetic energy of the atoms and their trapping in the optical potential, the atoms keep interacting with the light fields and this induces fluctuations in the atomic dynamics. Indeed, consider an atom that has already lost enough energy to be trapped at the bottom of a potential well. The atom will then oscillate at the bottom of the well. This is the situation illustrated in Fig. 8

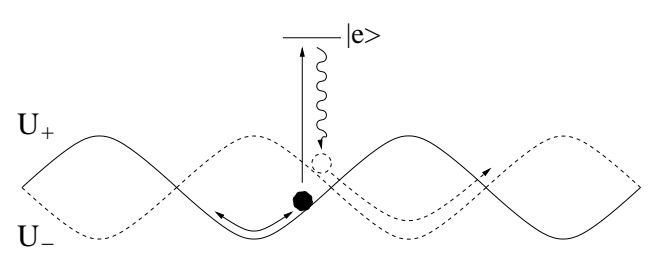

FIG. 8: Stochastic process of optical pumping transferring, via an excited state, an atom from a potential to the other one. The filled (empty) circle represents the atom in the $|g,+1 / 2\rangle(|g,-1 / 2\rangle)$ ground state sublevel.

To be specific, consider for example an atom initially in the $|g,+1 / 2\rangle$ state. Exactly at the center of the well the light polarization is purely $\sigma^{+}$, which does not allow the transfer from the $|g,+1 / 2\rangle$ state to the $|g,-1 / 2\rangle$ sublevel. However, out of the center of the well the light has also a nonzero $\sigma^{-}$component, which results in a nonzero probability to transfer the atom from its original sublevel to the other one. Therefore the atom can be transfered from one sublevel to the other one, and also the potential experienced by the atom will change from $U_{+}$to $U_{-}$, i.e. the force experienced by the atom will change. As optical pumping is a stochastic process, the (stochastic) transfer from a sublevel to the other one results in a fluctuating force. Figure 8 also shows how optical pumping between different optical potentials leads to the transport of atoms through the lattice: although the trapped atom does not have enough energy to climb the potential hill, optical pumping allows the transfer from a potential well to the neighbouring one. The optical pumping leads then to 
a random walk of the atoms through the optical potential, and indeed normal diffusion has been experimentally observed for an atomic cloud expanding in an optical lattice [32].

The last element necessary to implement a rocking ratchet is the oscillating force. In order to generate a time-dependent homogeneous force, one of the lattice beams is phase modulated, so that to obtain the electric field configuration:

$$
\frac{1}{2} E_{0}\left\{\vec{\epsilon}_{x} \exp [i(k z-\omega t)]+\vec{\epsilon}_{y} E_{0} \exp [i(-k z-\omega t+\alpha(t))]\right\}+\text { c.c. }
$$

where $\alpha(t)$ is the time-dependent phase. In the laboratory reference frame this laser configuration generates a moving optical potential $U[2 k z-\alpha(t)]$. Consider now the dynamics in the moving reference frame defined by $z^{\prime}=z-\alpha(t) / 2 k$. In this accelerated reference frame the optical potential is stationary. In addition to the potential the atom, of mass $m$, experiences also an inertial force $F$ in the $z$ direction proportional to the acceleration $a$ of the moving frame [33]:

$$
F=-m a=\frac{m}{2 k} \ddot{\alpha}(t) .
$$

In this way in the accelerated frame of the optical potential the atoms experience an homogeneous force which can be controlled by varying the phase $\alpha(t)$ of one of the lattice beams. The appropriate choice of the phase $\alpha(t)$ for the realization of the spatially symmetric rocking ratchet is

$$
\alpha(t)=\alpha_{0}\left[A \cos (\omega t)+\frac{B}{4} \cos (2 \omega t-\phi)\right]
$$

with $\phi$ constant. Indeed, by using Eq. 18, we can see immediately that in the accelerated frame of the optical potential the phase modulation $\alpha(t)$ will result into a force

$$
F=\frac{m \omega^{2} \alpha_{0}}{2 k}[A \cos (\omega t)+B \cos (2 \omega t-\phi)]
$$

which is of the form needed for the realization of the spatially symmetric rocking ratchet, as discussed in Sec. 【IID.

Experimentally, it is possible to obtain a phase modulation of the form (19) by simply using acousto-optical modulators and a set of radio-frequency generators. The exact technical realization is of no particular interest here, and we refer to Ref. [31] for further details. We only notice that it is possible experimentally to carefully control the phase difference $\phi$ between the two harmonics. This allows us to carefully control the symmetry of the system (see Sec. IIID). 


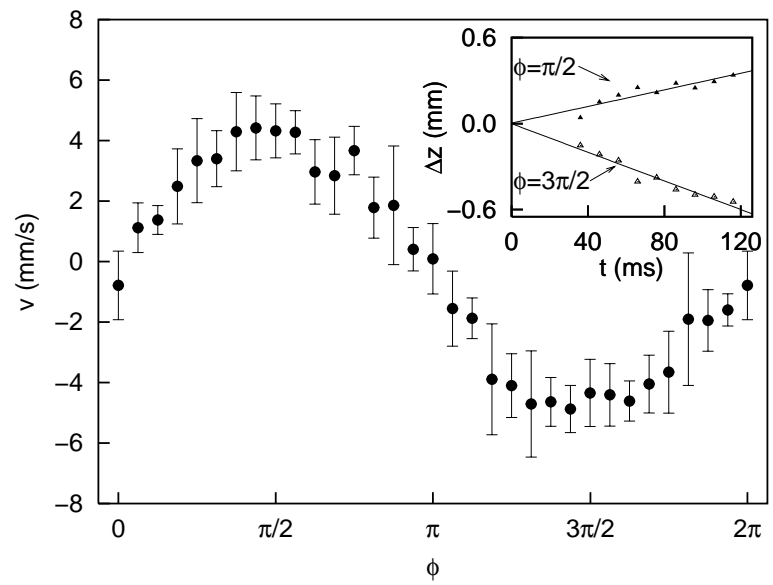

FIG. 9: Velocity of mass of the atomic cloud as a function of the phase $\phi$. Inset: displacement of the center of mass of the atomic cloud as a function of time for two different values of the phase $\phi$. Figure taken from Ref. [31].

The experiment of Ref. [31] clearly demonstrated the control of the current through a spatially symmetric potential by varying the time-symmetries of the system. In that work, the dynamics of the atoms in the optical lattice was studied by direct imaging of the atomic cloud with a CCD camera. For a given phase $\phi$ the position of the center of mass of the atomic cloud was studied as a function of time. It should be noticed that in principle it is necessary to transform the measurements from the laboratory reference frame to the accelerated reference frame of the optical potential, by using the coordinate transformation $z^{\prime}=z-\alpha(t) / 2 k$. However in the case of Ref. [31] this is not necessary as for the typical time scales of that experiment (period of the ac force and imaging time) the measured positions of the c.m. of the atomic cloud in the laboratory and in the accelerated reference frame are approximately equal. The results of that experiment are reported in Fig. 9, It can be seen that the center of mass of the atomic cloud moves with constant velocity (see inset). This velocity shows the expected dependence on the phase $\phi$ : for $\phi=n \pi$, with $n$ integer, the velocity (current of atoms) is zero, while for $\phi=\pi / 2,3 \pi / 2$ the velocity reaches a maximum (positive or negative). This because although the symmetry $F(t+T / 2)=-F(t)$ is broken for any value of the phase $\phi$, there is a residual symmetry $F(t)=F(-t)$ which forbids the current generation. This symmetry is controlled by the phase $\phi$ : for $\phi=n \pi$ it is realized, while for $\phi=(2 n+1) \pi / 2$ it is maximally broken. 
The experiment of Ref. [31] constitutes therefore a clear demonstration of the ideas of Sec. IIID the atoms can be set into directed motion through a symmetric potential by breaking the temporal symmetry of the system. We notice that the described experiment reproduces well the dependence of the current on the phase $\phi$ derived in Sec. IIID on the basis of the analysis of symmetries which apply in the Hamiltonian limit, i.e. in the absence of dissipation. This because the experiment of Ref. [31] was made in the regime of relatively strong driving, which well approximates the Hamiltonian regime. The role of the dissipation in a spatially symmetric system was examined in a subsequent investigation 34]. In that work the possibility to rectify fluctuations in a spatially symmetric optical lattice was also demonstrated, i.e. a Brownian motor was realized.

In order to discuss the role of fluctuations, it is essential first to introduce the quantity appropriate to describe the amplitude of the fluctuations in an optical lattice. We have previously seen that in an optical lattice fluctuations are determined by optical pumping between different ground state sublevels. Therefore the appropriate quantity to describe the amplitude of the fluctuations is the optical pumping rate, i.e. the number of optical pumping cycles per unit time.

In the work of Ref. 34] the current of atoms through the lattice was studied as a function of the applied ac force for different values of the optical pumping rate $\Gamma^{\prime}$. It should be notice that in an optical lattice the optical pumping rate can be varied while keeping constant the optical potential. Indeed for an intensity $I$ of the lattice beams, and a detuning $\Delta$ from atomic resonance, the optical pumping rate is proportional to $\Gamma^{\prime} \propto I / \Delta^{2}$ while the depth $U_{0}$ of the optical potential scales as $U_{0} \propto I / \Delta$. Therefore by changing simultaneously $I$ and $\Delta$ it is possible to keep $I / \Delta$ constant while varying $I / \Delta^{2}$, i.e. it is possible to change the optical pumping rate while keeping constant the optical potential. Experimentally it is possible to verify that the potential is constant by using pump-probe spectroscopy [35]. This technique indeed allows a precise measurement of the oscillation frequency at the bottom of the potential wells.

Figures [10 and 11] (from Ref. 34]) show the results of numerical simulations and experiments.

The experimental and numerical data are consistent and show a clear dependence on the amplitude of the applied force and on the optical pumping rate. Consider first the 


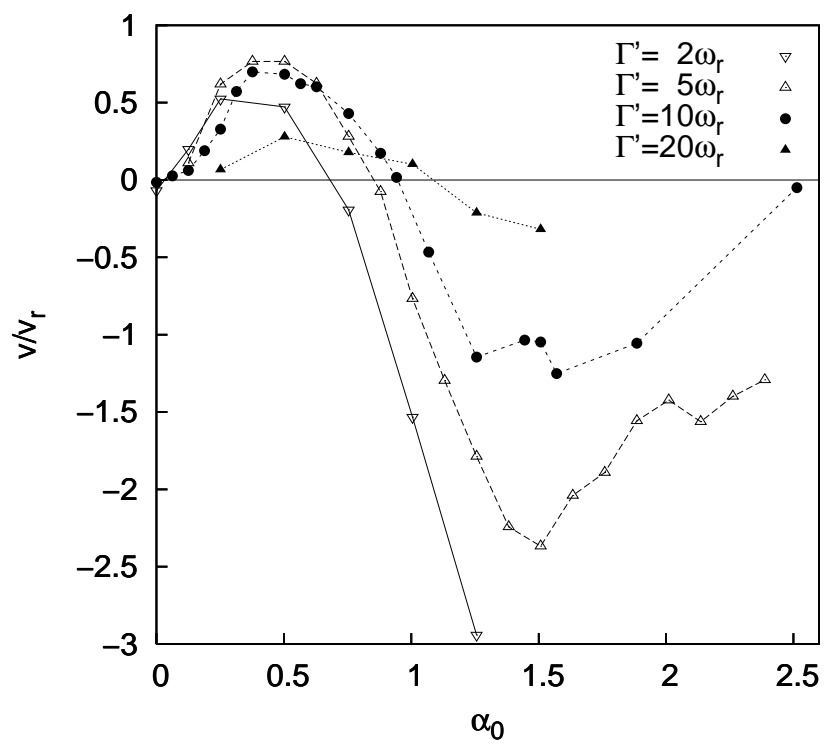

FIG. 10: Numerical simulations for a sample of atoms in a 1D lin $\perp$ lin optical lattice. The average atomic velocity, in units of the recoil velocity $v_{r}=\hbar k / m$, is shown as a function of the amplitude of the phase modulation. Different date sets correspond to different optical pumping rates $\Gamma^{\prime}$, expressed in terms of the recoil frequency $\omega_{r}=\hbar k^{2} / 2 m$. The optical potential is the same for all data. The relative phase between the two harmonics is $\phi=\pi / 2$, a choice which breaks the time symmetry of the system.

dependence on the ac force magnitude. For a small amplitude of the ac force the average atomic velocity is an increasing function of the force amplitude., with the atoms moving in the positive direction. At larger amplitude of the ac force the velocity decreases, and a current reversal is observed, with the atomic cloud moving in the negative direction. This kind of behaviour, named current reversal, is typical of rocking ratchets [5]. We examine now the dependence of the current on the optical pumping rate, i.e. on the noise level. We observe from Figs. 1011 that the dependence of the current on the optical pumping rate is very different depending on the ac force amplitude. For large amplitude of the applied force the magnitude of the current (in absolute value) is a decreasing function of the optical pumping rate. This means that in this regime the motion can be attributed to deterministic forces and correspond to force rectification by harmonic mixing: in a nonlinear medium the two harmonics, of frequency $\omega$ and $2 \omega$ and phase difference $\phi$, are mixed and the rectified 

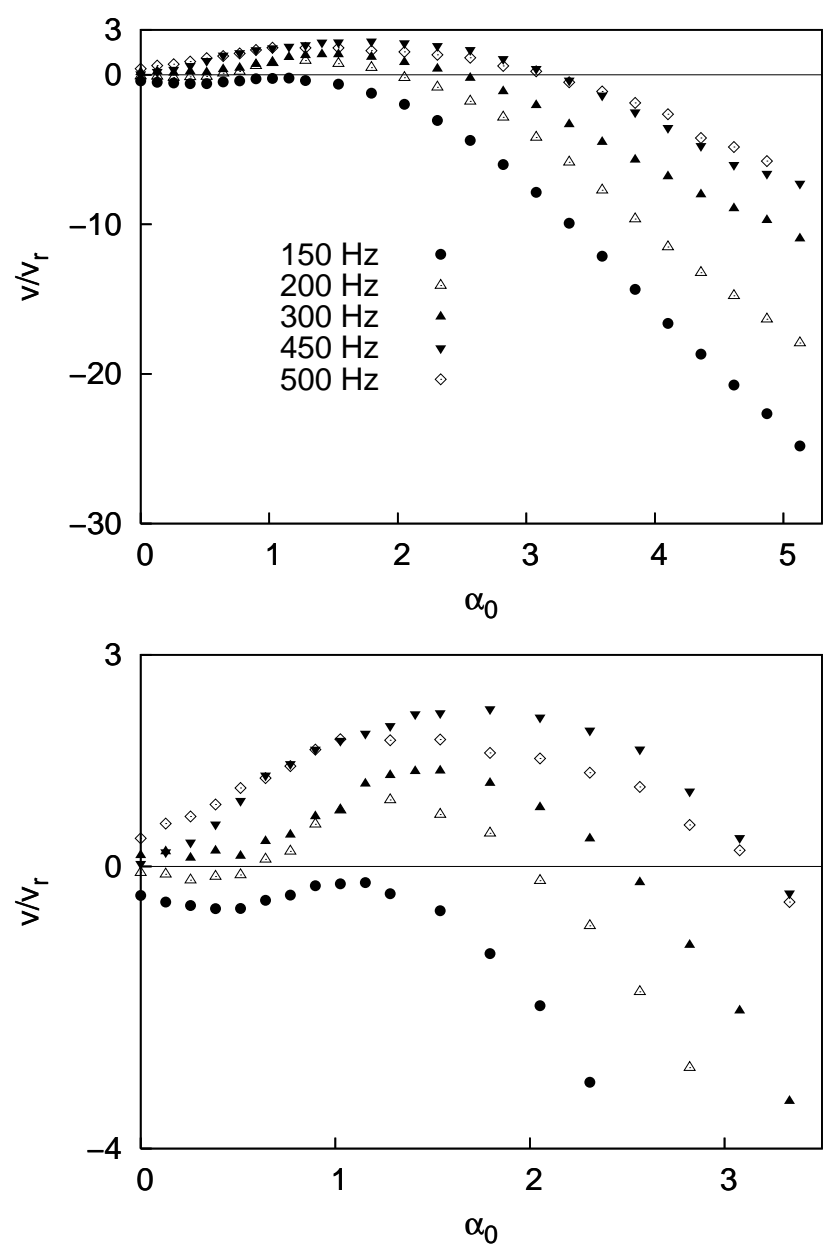

FIG. 11: Experimental results for the atomic velocity as a function of the amplitude of the phase modulation. The top graph include all experimental results, while the bottom graph evidences the region of small ac forces. The optical potential is the same for all measurements. Different data sets correspond to different optical pumping rate, and they are labeled by $\Gamma_{s}=\left[\omega_{v} /(2 \pi)^{2}\right] / \Delta\left(\omega_{v}\right.$ is the vibrational frequency) which is proportional to the optical pumping rate.

force produces a current $C \propto \sin \phi$, as discussed in Sec. IIID, In the considered experiment the nonlinearity of the medium is the anharmonicity of the optical potential. In this regime of rectification of the forces the noise does not play any constructive role in the generation of the current of atoms. On the contrary, the noise disturbs the process of rectification of the forces, and indeed in the current decreases for increasing optical pumping rate. We conclude that this regime does not correspond to the rectification of fluctuations. A very different 
dependence of the current amplitude on the optical pumping rate is found in the regime of small amplitudes of the applied force. Indeed in this regime the current is for small pumping rates an increasing function of the pumping rate, and the current vanishes in the limit of vanishing optical pumping rates. At larger pumping rates the current reaches a maximum and then decreases again. This bell-shaped dependence of the current on the optical pumping rate is a typical signature of a Brownian motor: in the absence of fluctuations the current is zero, then increases until the fluctuations are so large that the presence of the potential and of the applied fields become irrelevant, and the current decreases again. We can therefore conclude that in the regime of small ac force amplitude the optical lattice provides an implementation of a Brownian motor.

\section{CONCLUSIONS}

In this review the basic ideas underlying Brownian motors have been outlined and two different implementations, the flashing ratchet and the rocking ratchet, described. The important role played by the symmetries in determining a current has been pointed out. The second part of this review discusses a recent implementation of the rocking ratchet using cold atoms in an optical lattice. Optical lattices are an ideal model system for statistical physics because of their tunability: both the optical potential and the noise level can be carefully controlled. The described experiment shows well the rectification of fluctuations, and therefore demonstrates the realization of a Brownian motor with cold atoms in an optical lattice.

The aim of the present review is also to give an idea of the important role that optical lattices can play as model system to study phenomena of statistical physics. This goes beyond the modelling of Brownian motors. For example the phenomenon of stochastic resonance has been recently observed in a near-resonant optical lattice [36, 37]. Far-detuned optical lattice have been used to investigate chaotic motion, both in the classical and quantum regime [38]. Finally, a new class of ratchets has been introduced which combines "standard" ideas of thermal ratchets with chaos: these are deterministic chaotic ratchets in which there are no thermal fluctuations and it is chaos which mimics the role of noise [39]. It is not difficult to predict that optical lattices will play a major role in the experimental study of this new class of ratchets. 


\section{Acknowledgments}

The author is grateful to EPSRC, UK and to the Royal Society for financial support, and to Eric Lutz for critical reading the manuscript.

[1] Brown, R., 1828, Phylos. Mag., 4, 161.

[2] Einstein, A., 1905, Ann. Phys. (Berlin), 17, 549.

[3] von Smoluchowski, M., 1906, Ann. Phys., 21, 756.

[4] Langevin, P., 1908, Comptes Rendues, 146, 530.

[5] Reimann, P., 2002, Phys. Rep., 361, 57.

[6] Astumian, R.D., 1997, Science, 276, 917.

[7] Bier, M., 1997, Contemp. Phys., 38, 371.

[8] McClintock, P.V.E., 1999, Nature, 401, 23.

[9] Astumian, R.D., and Hänggi, 2002, Phys. Today, 55, 33.

[10] Parrondo, J.M.R., and Dinis, L., 2004, Contemp. Phys., 45, 147.

[11] Jülicher, F., Ajdari, A., and Prost, J., 1997, Rev. Mod. Phys., 69, 1269.

[12] Linke, H., Sheng, W., Löfgren, A., Xu, H., Omling, P., and Lindelof, P.E., 1998, Europhys. Lett., 44, 341.

[13] Linke, H., Humphrey, T.E., Löfgren, Sushkov, A.O., Newbury, R., Taylor, R.P., Omling, P., 1999, Science, 286, 2314.

[14] Weiss, S., Koelle, D., Müller, J., Gross, R., and Barthek, K., 2000, Europhys. Lett., 51, 499.

[15] Feynman, R.P., Leighton, R.B., and Sands, M., The Feynman Lectures on Physics (Addison Wesley, Reading MA, 1963).

[16] Brillouin, L., 1950, Phys. Rev. A, 78, 627.

[17] Adjari, A., and Prost, J., 1993, CR Acad. Sci. (Paris) II, 315, 1635.

[18] Magnasco, M.O., 1993, Phys. Rev. Lett., 71, 1477.

[19] Bartussek, R., Hänggi, P., and Kissner, J.G., 1994, Europhys. Lett., 28, 459.

[20] Goychuk, I., and Hänggi, P., 1998, Europhys. Lett., 43, 503.

[21] Flach, S., Yevtushenko, O., and Zolotaryuk, Y., 2000, Phys. Rev. Lett., 84, 2358.

[22] Yevtushenko, O., Flach, S., Zolotaryuv, Y., and Ovchinnikov, A.A., 2001, Europhys. Lett.,54, 
141.

[23] Denisov, S., Flach, S., Ovchinnikov, A.A., Yevtushenko, O., and Zolotaryuk, Y., 2002, Phys. Rev. E,66, 041104.

[24] Dykman, M.I., Rabitz, H., Smelyanskiy, and Vugmeister, B.E., 1997, Phys. Rev. Lett.,70, 1178.

[25] Reimann, P., 2001, Phys. Rev. Lett.,86, 4992.

[26] Mennerat-Robilliard, C., Lucas, D., Guibal, S., Tabosa, J., Jurczak, C., Courtois, J.-Y., and Grynberg, G., 1999, Phys. Rev. Lett., 82, 851.

[27] Meacher, D.R., 1998, Contemp. Phys., 39, 329.

[28] Guidoni, L., and Verkerk, P., 1999, J. Opt. B: Quantum Semiclass. Opt., 1, R23.

[29] Grynberg, G. and Mennerat-Robilliard, C., 2001, Phys. Rep., 355, 335.

[30] Dalibard, J., and Cohen-Tannoudji, C., 1989, J. opt. Soc. Am., B6, 2023; Ungar, P.J., Weiss, D.S., Riis, E., and Chu, S., 1989, J. opt. Soc. Am., B6, 2058.

[31] Schiavoni, M., Sanchez-Palencia, L., Renzoni, F., and Grynberg, G., 2003, Phys. Rev. Lett., 90, 094101.

[32] Carminati, F.R., Schiavoni, M., Sanchez-Palencia, L., Renzoni, F., and Grynberg, G., 2001, Eur. Phys. J. D, 17, 249.

[33] Landau, L.D., and Lifshitz, E.M., Mechanics (Pergamon Press, Oxford, 1976).

[34] Jones, P.H., Goonasekera, M., and Renzoni, F., 2004, Phys. Rev. Lett., 93, 073904.

[35] Carminati, F.-R., Schiavoni, M., Todorov, Y., Renzoni, F., and Grynberg, G., 2003, Eur. Phys. J. D, 22, 311.

[36] Sanchez-Palencia, L., Carminati, F.-R., Schiavoni, M., Renzoni, F., and Grynberg, G., Phys. Rev. Lett., 88, 2002, 133903.

[37] Schiavoni, M., Carminati, F.-R., Sanchez-Palencia, L., Renzoni, F., and Grynberg, G., 2002, Europhys. Lett., 59, 493.

[38] Raizen, M.G., Philos. Mag. B, 2000, 90, 2109.

[39] Jung, P., Kissner, J.G., and Hänggi, P., 1996, Phys. Rev. Lett., 76, 3436.

\section{Author's biography}


Ferruccio Renzoni studied Physics at the University of Pisa (Italy) where he obtained his M.Sc. in 1993. He then obtained his Ph.D. from the Technische Universität Graz (Austria) in 1998 with a thesis on coherent population trapping in atomic systems. He then spent two years in Germany, at the Institut für Laserphysik of the University of Hamburg, and three years in France, at the Laboratoire Kastler Brossel (Ecole Normale Supérieure, Paris), where he obtained his Habilitation à diriger des recherches. During the stay in Germany and France, he became interested in optical lattices and in the possibility to use them to generate models for statistical mechanics. Since 2003 he is at the Department of Physics and Astronomy of the University College London, where he leads the laser cooling group. 